for the period July 1, 1989 to June 30, 1993

Grant No. DE-AI02-89ER14068.A001

R. H. Atalla

USDA Forest Products Laboratory

Madison, Wisconsin 53705-2398

\title{
DISCLAIMER
}

This report was prepared as an account of work sponsored by an agency of the United States Government. Neither the United States Government nor any agency thereof, nor any of their employees, makes any warranty, express or implied, or assumes any legal liability or responsibility for the accuracy, completeness, or usefulness of any information, apparatus, product, or process disclosed, or represents that its use would not infringe privately owned rights. Reference herein to any specific commercial product, process, or service by trade name, trademark, manufacturer, or otherwise does not necessarily constitute or imply its endorsement, recommendation, or favoring by the United States Government or any agency thereof. The views and opinions of authors expressed herein do not necessarily state or reflect those of the United States Government or any agency thereof. 
We have previously shown that all relatively pure plant and bacterial celluloses are, in their native states, composites of two lattice forms, $I_{\alpha}$ and $I_{\beta}$, and that the two forms possess molecular chains in the same conformations but with different hydrogen bonding patterns. In the current period we have demonstrated that in higher plant cell wall matrices, the hemicelluloses are likely to have a regulatory function during the aggregation of cellulose. Different hemicelluloses appear to influence the aggregation in different ways. We have also developed preliminary evidence indicating the hemicelluloses may have a protective function against the action of some cellulolytic enzymes.

Our earlier studies using the Raman microprobe had shown that lignin is more highly organized in the cell wall than had theretofore been recognized. In the current period we have developed further the foundation for more detailed mapping of the variability of lignin within cell wall structures. To do so we have advanced our understanding of the contribution of the different lignin substructures to the intensity of the most characteristic of the lignin Raman bands which stands apart from the region where the polysaccharide matrix components contribute to the spectra. We have also developed preliminary evidence of associative interactions between the precursors or oligomers of lignin and the polysacchride matrix, and that these associative forces may cause the polysaccharides to function as a template in the organization of lignin precursors prior to polymerization. We have also demonstrated that electron transfer pathways are present within the structure of native lignins; we believe these pathways may be the key to extension of the action of some of the oxidizing enzymes into domains from which the enzymes may be excluded because of their size.

The specific accomplishments during the current period are:

Demonstration that hemicelluloses present during biogenesis can transform bacterial cellulose into a cellulose typical of higher plant celluloses, and that each hemicellulose has a different effect on the pattern of aggregation.

Preliminary evidence that the hemicelluloses may limit the action of certain cellulolytic enzymes, suggesting that their function may go beyond regulation to include passive resistance to cellulolytic pathogens.

Enhancing the potential of the Raman microprobe technique for mapping variability of lignin in the cell wall by identifying the contribution of the different substructures of lignin to the intensity of the key band in the Raman spectrum of lignin.

Mapping of the variability of lignin across two cell wall sections. The mappings have convoluted within them both composition and concentration, but they demonstrate the potential of the method and point to the improvements we are now making so as to distinguish between variability of concentration and variability of composition. 
Establishing the structure and molecular orientation in spiral thickening on the interior lumen walls of Taxus brevifolia tracheids.

Conclusive evidence for the presence of electron transport pathways within the structure of native lignin, and preliminary evidence concerning the dependence of these pathways on structure.

Preliminary experimental evidence that the polysaccharide matrix may provide the primary organizing framework for the order that is observed in lignin.

Molecular modeling studies that provide insight into the association of both oligolignins and polysaccharide oligomers with surfaces of ordered cellulose domains. 


\begin{abstract}
MOLECULAR ORGANIZATION IN THE NATIVE STATE OF WOODY TISSUE: STUDIES OF TERTIARY STRUCTURE USING THE RAMAN MICROPROBE SOLID STATE 13C NMR AND BIOMIMETIC TERTIARY AGGREGATES
\end{abstract}

\title{
INTRODUCTION
}

The period covered by the present report has been transitional in a number of respects. The most significant one has been the shift of the perspective from one with an emphasis on studies of structure in the native state to one wherein inquiry into the processes of aggregation and integration of constituents into the the cell wall matrix has become an essential part of the investigation.

The program was started eleven years ago when the prevailing paradigm for cell wall structure was that reflected in the treatises by Preston (1) and Frey-Wyssling (2) where the cell wall was portrayed as a two phase composite structure with the cellulose fibrils regarded as a relatively homogeneous anisotropic reinforcing structure, and the rest of the matrix was seen as an amorphous blend of hemicelluloses and lignin.

Since that time, our solid state 13C NMR studies, in collaboration with Dr. David L. VanderHart of NIST, have shown that all native celluloses are composites of two lattice forms $I_{\alpha}$ and $I_{\beta}$, with the $I_{\alpha}$ form dominant in bacterial and most algal celluloses, and the $I_{\beta}$ form dominant in the higher plant celluloses (3-5), and our Raman spectral investigations of these two forms have shown them to possess cellulose chains with similar conformations but with different hydrogen bonding patterns $(6,7)$. During the current period we have further shown that the presence of the hemicelluloses has a significant influence on the patterns of aggregation of the cellulose and have proposed the hypothesis that one of their functions may indeed be the regulation of the aggregation of the cellulose in the cell wall matrix $(8-10)$. We have also initiated an exploration of the influence of hemicelluloses on the susceptiblity of the cell wall matrix to cellulolytic enzymes in collaboration with Drs. Liisa Viikari and Tuula Teeri and their coworkers at the Biotechnical Laboratory of the Technical Research Center of Finland (VTT).

Our work using the Raman microprobe has shown that lignin is more highly organized within the cell wall matrix than had theretofore been recognized, and that there is some correlation between its organization and that of the cellulose within the plane of the cell wall (11-13). During the current period we have developed a deeper understanding of the manner in which structural variability influences the intensity variations of the characteristic bands of lignin and have laid the foundation for the broader mapping studies which have long been one of our primary goals.

Because of the apparent correlation between the organization of cellulose and lignin in the cell walls, we have initiated explorations of possible associative interactions between the constituents of lignin and the polysaccharide matrix. In preliminary spectral measurements as well in 
molecular modeling calculations we have found indications that such interactions can occur. We have also initiated a cooperative effort with Professor N. Terashima, Emeritus of Nagoya University and currently in our laboratory as a visiting scientist, to inquire into the mechanisms involved in the integration of lignin within the polysaccharide matrix. Preliminary results of these explorations are described in subsequent sections of this report.

Perhaps the greatest departure from past paradigms and methodologies is our exploration into the electron transport characteristics of the lignified cell wall matrix. It grew out of our awareness that if pathways for electronic charge transport were available, they could provide additional insight concerning the organization of the lignin since conductivity in polymeric structures is quite sensitive to short and intermediate range order. Our studies of photoconductivity to date, described in outline in a subsequent section, suggest that such measurements may indeed be developed into a very useful probe of the organization of lignin in the native state.

The other transitions alluded to above include completion of the relocation of our program from the Institute of Paper Chemistry to the USDA Forest Service Forest Products Laboratory and the University of Wisconsin in Madison. They also include the expansion of the possibilities of pursuing a broader perspective with respect to research in related areas of plant science; such pursuits had been precluded in the prior context with its emphasis on plant resources that are relevant to the pulp and paper industries.

\section{THE POLYSACCHARIDE MATRIX}

The realization that native celluloses are composites of two lattice forms of cellulose naturally led to questions about the processes through which such composites could arise, and how these could be reconciled with what is known about the biogenesis of cellulose from Acetobacter xylinum, which is the organism that produces the highest levels of the $I_{\alpha}$ lattice form. In reviewing the discussions by Haigler (14) and by Haigler and Benziman (15) of the assembly of cellulose microfibrils, with the knowledge that the key difference between the two lattice forms is the pattern of hydrogen bonding. it occurred to us that the aggregation of chains within the bundle emerging from a single assembly complex could have one hydrogen bonding pattern, while the aggregation between the bundles emerging from adjacent assembly complexes could result in a different hydrogen bonding pattern. Thus, we would view the single crystalline domains observed by Sugiyama et al, in their lattice imaging studies (16), as superlattice structures, with the relationship of the $I_{B}$ domains to each other defined by the superlattice. This possibility is not excluded by the lattice images because these images depend on electron scattering by the heavy atoms, and would not be sensitive to the differences in hydrogen bonding, which is the primary difference between the two forms of cellulose $I$.

The accumulated evidence that the aggregation pattern could be altered by the presence of agents that can associate with cellulose $(15.17)$. suggested that the process of biogenesis must include a stage of nascence during which this pattern of aggregation of the bundles emerging from individual assembly complexes could be modified. This led us to the speculation that, if the 
assembly mechanism in higher plants is similar to that in Acetobacter xylinum, some of the constituents of the cell wall matrix could have similar ef fects.

Our inquiry into this matter has unfolded in two phases described in Attachment 1. The early phase was based on procedures required to carry out meaningful experiments using the commonly available strains of Acetobacter xylinum and had inherent in it severe limitations on the amount of modified cellulose that could be produced. A more detailed description of the results of the first phase is given in Attachment 2, which will be submitted for publication in the near future. The second phase has taken advantage of the more recently available agitation tolerant strain of the organism, and has hence made possible production of modified celluloses in quantities which allow acquisition of SS 13C NMR spectra using the CP/MAS technique. The results based on the NMR studies are described in Attachment 3, which will be submitted for publication within the month. A more detailed account including results on two additional hemicelluloses is currently in preparation.

The methods used to characterize the celluloses during the first phase included electron microscopy, $x$-ray diffractometry, Raman spectroscopy, and the application of chemical analyses for sugar content determination. The key conclusions of the work are that the hemicelluloses can indeed modify the patterns of aggregation of the cellulose, and, perhaps equally important, each of the hemicelluloses modifies the aggregation in a different manner.

The methods used in the second phase included all but the electron microscopy, but added the application of SS 13C NMR to characterize the celluloses because the samples could be prepared in amounts sufficient for the acquisition of such spectra. The results confirmed the earlier findings that each of the hemicelluloses had a distinctive effect on the pattern of aggregation of the cellulose. In addition, however, they included the very significant observation that, with respect to the balance between $I_{\alpha}$ and $I_{\beta}$ lattice forms. the hemicelluloses shifted it so that it was much more like the higher plant celluloses than those of the control culture. This was one of the findings which led us to propose that the hemicelluloses may indeed function as regulators of the aggregation of cellulose during the formation of the cell wall matrix $(8-10)$.

Other important observations made in the second phase, to be described in the later report, include the finding that the hemicelluloses are intimately integrated into the stucture of the cellulose. The usual isolation procedure includes boiling, under nitrogen, in $1 \% \mathrm{NaOH}$ solution, which would remove any material adsorbed on the surface. This interpretation was further tested by extracting the glucomannan modified cellulose in $17.5 \% \mathrm{NaOH}$ solutions; only one third of the hemicellulose incorporated could be removed by this extraction. Our conclusion from these observations is that the hemicelluloses may well be entrapped between the bundles emerging from individual assembly complexes.

Yet another part of our characterization of the modified bacterial celluloses, which model the polysaccharide cell wall matrix, has been an exploration of their susceptibility to the action of well characterized cellulolytic enzymes isolated from the fungus. Trichoderma reesi. This has been carried out in cooperation with the group of Dr. Lilsa Vilkarl at VTT who have isolated highly purified fractions of many of the enzymes produced by this organism. 
This work is at an early phase, but it has raised new questions about functions of hemicelluloses that may have been overlooked. The preliminary observations on the mannan and glucomannan modified bacterial celluloses is that they were resistant to the action of an endoglucanase and a cellobiohydrolase which were applied to them, except when a mannanase was added to the mixture of enzymes. While these results are in need of further confirmation, and some additional complementary experiments must be carried out, they do raise the possibility that the hemicelluloses may play a role in defense of the organism by binding with, and limiting the action of the enzymes which are specific to cellulose. We are actively pursuing this line of inquiry because it also speaks to the issue of the intimacy of integration of the constituents at the nanoscale level.

The findings we have reported on the polysaccharide matrix are a key component of the data that have led us to recognize the need to re-examine the accepted paradigms concerning cell wall structure. As Haigler (14) and Haigler and Benziman (15) have noted, the bundles emerging from individual assembly complexes are approximately $1.5 \mathrm{~nm}$ in diameter. They would thus contain no more than 13 molecular chains, would suffice to populate only four unit cells in the cross section, and would have more chains at the surface than within the bundle. They would also no longer meet one of the key criteria for being regarded as a separate phase, set forth most precisely by Landau and Lifshitz (16). as the requirement that the amount of matter at the surface be vanishingly small relative the amount of matter within the boundary. It is for this reason that we belleve the models of the cell wall matrix are in need of re-assessment. In particular, there is a need for models which acknowledge the high level of organization within the cell wall at a heirarchy of levels, without forcing the data to fit into a conceptual framework that has implicit in its definitions the characteristics of multidomain systems consisting of internally homogeneous individual domains.

A deeper understanding of the polysaccharide matrix is essential not only because of the complex and key role it plays in the processes of the cell, but also because it is the context within which the processes of lignification occur. Thus, though in the early stages of our work we were pursuing studies of structure with respect to cellulose and lignin along relatively independent paths, these paths have now converged in a natural and logical manner.

\section{LIGNIN AND LIGNIFICATION}

Our interest in the structure of lignin initially arose as our inquiry into the cell wall polysaccharides was extended to include the native state. It seemed unlikely to us that all other constituents of the cell wall would be highly organized and that the lignin, in contrast, would be a "random amorphous polymer". It occurred to us that the then-current views about the organization of lignin reflected the lack of reliable methods to detect such organization if it were present. This led us to the proposal to develop the application of the Raman microprobe as a device to meet this need. Our early studies demonstrated that lignin is indeed organized with respect to the plane of the cell wall, and demonstrated a variability within the secondary wall between regions that might have otherwise been considered equivalent $(11-13)$. 
The acquisition of the Raman microprobe spectra was so time consuming. however, that with the spectrometer system available at the time it would not have been feasible to consider the mapping studies that seemed the most logical extension of our early findings. We sought and received support from the DOE University Research Instrumentation Program for the development of a new microprobe that would utilize diode array detection systems to allow acquisition of spectra in sufficiently short periods of time that mapping of variations within and across cell walls, and between adjacent cells, would be feasible. Though the system components were acquired in 1987, a number of serious problems with respect to the mechanical stability of the system set back our effort to begin the mapping.

When the mechanical stability problems were resolved, it became clear that we needed to develop a deeper understanding of the different contributions to the intensity of the dominant band in the Raman spectrum of lignin, which was the one used to monitor both concentration and orientation. The work was part of the foundation effort in the dissertation of J. S. Bond, and is the subject of Attachment 4. The key findings were that cesidual coniferyl alcohol and coniferaldehyde structures contributed disproportionately to the intensity of the band through conjugation and pre-resonance enhancement effects.

Because of the possibility of monitoring the evolution of the spectra in time it was also observed that the band intensity had a transient component when the sample sections were immersed in water or dueterium oxide during spectral acquisition; this is our technique for quenching the background fluorescence. This required that comparisons in our preliminary mapping studies be based on acquisitions of equal time duration at all points. A mapping acquired in this mode is shown in Figure 1. It shows the variation of the intensity of the Raman band at $1600 \mathrm{~cm}-1$ over an area 25 micrometers in length and 15 micrometers wide in a longitudinal section of two adjacent loblolly pine cells. Though the intensities shown in this three dimensional presentation have convoluted into them the variability of both composition and concentration, they show the power of this technique to reveal variability both within the cell walls and between adjacent cells. These mapping studies, which also were part of the dissertation of J. S. Bond, will be described in a report currently in preparation. An overview of recent progress in our Raman spectral studies is provided in Attachment 5 .

We have since established that, when tissue is immersed in water, the coniferaldehyde component undergoes a photochemically induced degradation process when it is exposed to the green excitation laser light at highly focused intensities. Reduction with sodium borohydride has stabilized the spectral features so that we expect to be able to distinguish the contributions of the different structural features to the primary band in future mapping studies. We are indeed currently in the process of acquiring spectra that are stable in time and will allow us to do the mappings.

One of the key questions arising from our findings conceming the organization of lignin relates to the mechanisms by which this organization is developed. We are pursuing these questions in collaboration with Professor Terashima. One of the approaches we have adopted is to polymerize lignin precursors within a polysaccharide matrix prepared by delignifiying wood sections under mild conditions. The Raman microprobe spectra of lignin analog polymers 
prepared in this way show a preferential orientation of the aromatic rings in alignment with the cellulose within the cell walls.

We have also initiated studies of the associative forces between lignin oligomers and the polysaccharide matrix using the BIOSYM molecular modeling programs. Our computations so far have shown that the net interactions of lignin oligomers with the 110 surface of a cellulose aggregate are associative. This finding reinforces our earlier point about the need for new perspectives with respect to nanoscale structures, because on the basis of traditional ideas about the hydrophilicty of cellulose and hydrophobicity of lignin one would not have anticipated an associative interaction. We expect to expand this component of our effort particularly as access to one of the DOE supercomputers with a BIOSYM license is expected in the near future.

In an interesting application of our Raman microprobe techniques to the study of woody tissue we have investigated the spiral thickenings on the interior of the lumen of walls of cells from Taxus brevifolia (Pacific yew). This species has been under investigation in our laboratory at FPL because of its harvest for production of taxol, an antitumor agent. Though these thickenings have been noted by wood anatomists on the basis of microscopic examination, their constitution and function were not known. The Raman microprobe spectra show them to contain a highly oriented cellulosic component and a significant amount of lignin. The most interesting aspect of these results is that the cellulose in the spiral thickenings appears to be oriented parallel to the axis of the tacheids rather than correlated with the geometry of the spiral thickenings themselves.

\section{ELECTRON TRANSPORT IN LIGNIFIED TISSUE}

Our interest in exploring pathways for electron transport within the structure of lignin was stimulated by our search for a better understanding of organization at the nanoscale level. Polymeric conductors are known to have charge transfer characteristics which are sensitive to order at the oligomeric level (19). Even though lignin does not have extended conjugation, which is usually thought of as a prerequisite for conduction in polymeric materials, its aromatic centers are separated by distances of the order of $0.5 \mathrm{~nm}$, and electronic charge transport between centers as far apart as 1.0 to $1.5 \mathrm{~nm}$ had been reported in other biological systems (20). We initially thought we would have to resort to the use of electron donor dopants to explore this possibility. However, upon further consideration it seemed possible that one or another of the electronic transitions associated with absorption by lignin in the ultraviolet region of the spectrum might have an excited state wherein the electrons were weakly bound, so they would have sufficient mobility to test our hypothesis that pathways for electron transport do in fact occur in lignin.

The measurements we have made so far have confirmed this speculation. Figure 2 shows the wavelength dependence of the pattern of photoconductivity in longitudinal sections of Douglass fir wood. The results are presented the dependence on excitation wavelength of the ratio of photocurrent to dark current under otherwise identical conditions. The figure shows data for untreated tissue in comparison with a control sample that had been treated in acid for an extended period of time and another that had been subjected to an 
acid chlorite treatment for 30 minutes at 70 degrees $C$. The acid treated sample was to test whether the high levels of divalent $\mathrm{Mn}$ ions, typical in the secondary walls of conifers, were contributing to this effect by functioning as electron donors; they are frequently used as dopants in inorganic systems. The acid chlorite treatment was to test whether the aromatic centers responsible for enhancement of the Raman band at $1595 \mathrm{~cm}-1$ were assoclated with the photoconductivity. These centers are attacked quite early in an acid chlorite treatment and result in significant intensity reduction before much delignification is accomplished. Clearly these centers, which we believe to be the residual coniferaldehyde substructures, contribute significantly to the photconductivity since it is reduced by more than an order of magnitude after the acid chlorite treatment.

Though we first observed the phenomenon of photoconductivity over one year ago, we have been reluctant to submit a report for publication until we were confident that we could rationalize our observations and that they were not artifacts. The measurements are difficult because they have to be made on samples which are sufficiently dry to limit lonic conductivity in the tissue, and the electrical currents are extremely low. We are now confident of the observations and expect to submit a report in the near future.

An obvious question, of course, is whether such pathways are simply coincidental to the order, or whether they play a role in the processes of the cell. One possibility which must be considered is that the pathways for electron transport could extend the domain of influence of some of the oxidative enzymes which play significant roles both in the polymerization of lignin during biogenesis, and in the depolymerization reactions induced by lignin degrading organisms. 


\section{REFERENCES}

1. Preston, R. D., "The Physical Biology of Palnt Cell Walls", Chapman and Hall, London, 1974.

2. Frey-Wyssling. A., "The Plant Cell Wall", Gebruder Borntrager, Berlin, 1976.

3. Atalla, R. H. and VanderHart, D. L. Science 223, 283 (1984).

4. VanderHart, D. L. and Atalla, R. H., Macromol. 17, 1465, (1984).

5. VanderHart, D.L. and Atalla, R.H., in "The Structure of Cellulose," R.H.Atalla, Ed., ACS Symp. Ser. 340, American Chemical Society, Washington, DC, p. 88 .

6. Wiley, J. H. and Atalla, R. H., in "The Structures of Cellulose", R. H. Atalla, Ed., ACS Sypm. Ser. 340. American Chemical Society, Washington, DC, 1987. p. 151.

7. Atalla, R. H. and VanderHart, D. L., in "Cellulose and Wood: Chemistry and Technology", C. Schuerch, Ed., Wiley-Interscience, New York, 1989, p. 169.

8. Atalla, R. H., Hackney, J. M., Uhlin, I, and Thompson, N. S., Int, J. Biol. Macromol. 15. 109(1993). Attachment 1 to Progress Report.

9. Uhlin, I, Atalla, R.H., and Thompson, N.S., (to be published). Attachment 2 to Progress Report.

10. Hackney, J. M., VanderHart, D. L., and Atalla, R. H., (to be published) Attachment 3 to Progress Report.

11. Atalla, R.H. and Agarwal, U.P., Science 227. 636(1985).

12. Agarwal, U.P. and Atalla, R.H., Planta 169, 325(1986).

13. Atalla, R.H. and Agarwal, U.P., J. Raman Spectroscopy 17, 229(1986).

14. Haigler, C. H., in "Biosynthesis and Biodegradation of Cellulose", C. H. Haigler and P. J. Weimer. Eds., Marcel Dekker, New York, 1991, p. 89.

15. Haigler, C. H. and Benziman, M., In "Cellulose and Other Natural Polymer systems", R. M. Brown, Ed., Plenum, New York, 1982, p. 273.

16. Sugiyama, J., Harada, H., Fujiyoshi, Y., and Uyeda, N., Mokuzai Gakkaishi, 31. 61(1985)

17. Kai, A. and Koseki, T., Chemistry Letters, The Chemistry Scoiety of Japan, 1985, p. 607 .

18. Landau, L. D. and Lifshitz, E. M. "Statisitical Physics", Addison Wesley, Pergamon, London, 1958, p. 5. 
19. Meyer, H., "Organic Semiconductors", Verlag Chemie, Weinheim, 1974.

20. Beratan, D. N., Onuchic, J. N., Winkler, J. R. and Gray, H. B., Science 258. $1740(1992)$. 


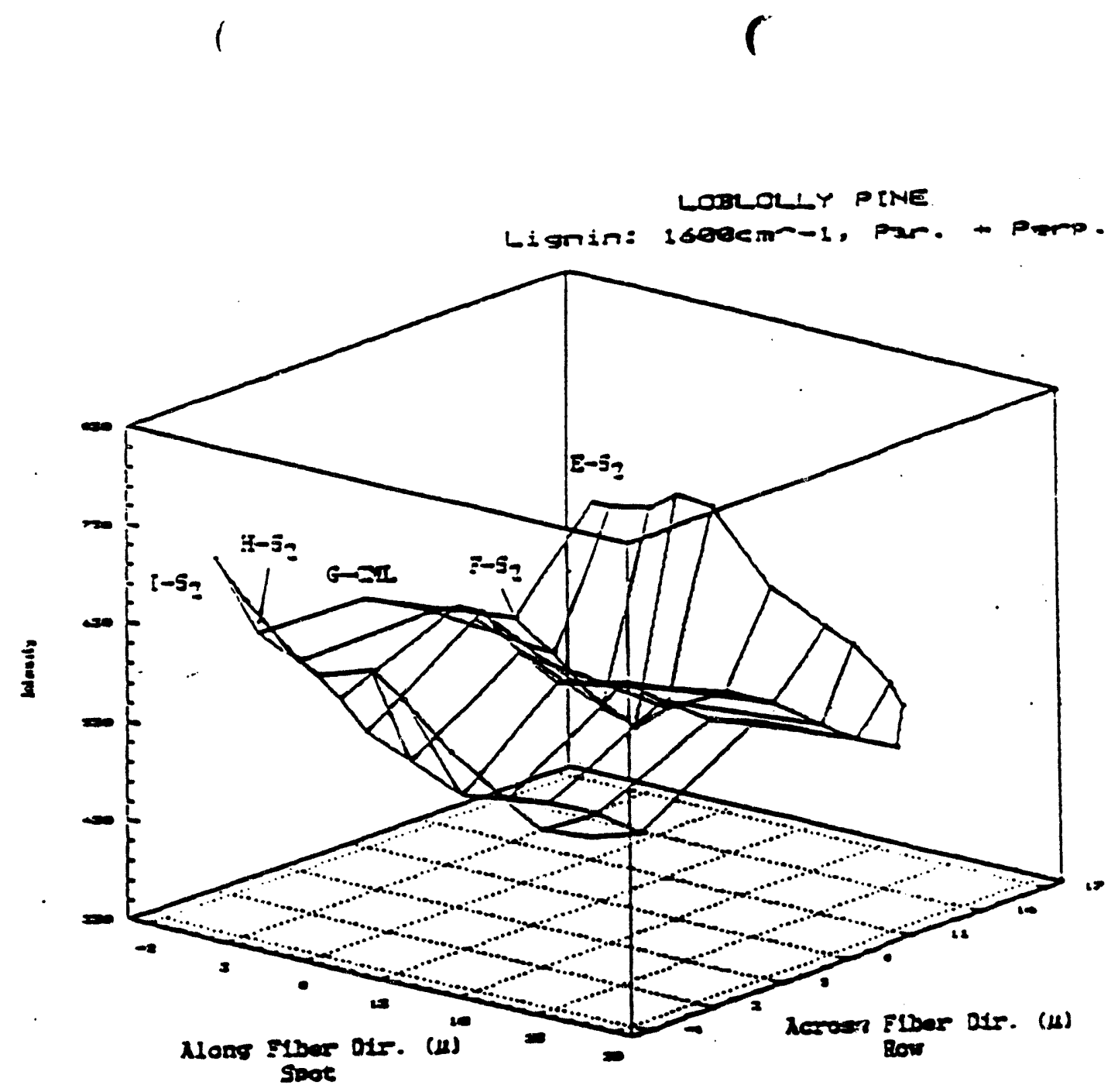

Figure 1. Topographical variation of the intensity of the $1600 \mathrm{~cm}^{-1}$ band in a longitudinal cross-section of two adjacent cell walls. The intensity messurenent consists of the sumation of the intensities measured with Blectric Vectors of the exciting laser parallel and perpendicular to the cell wall. 
Photoconductivities of samples with different treatments

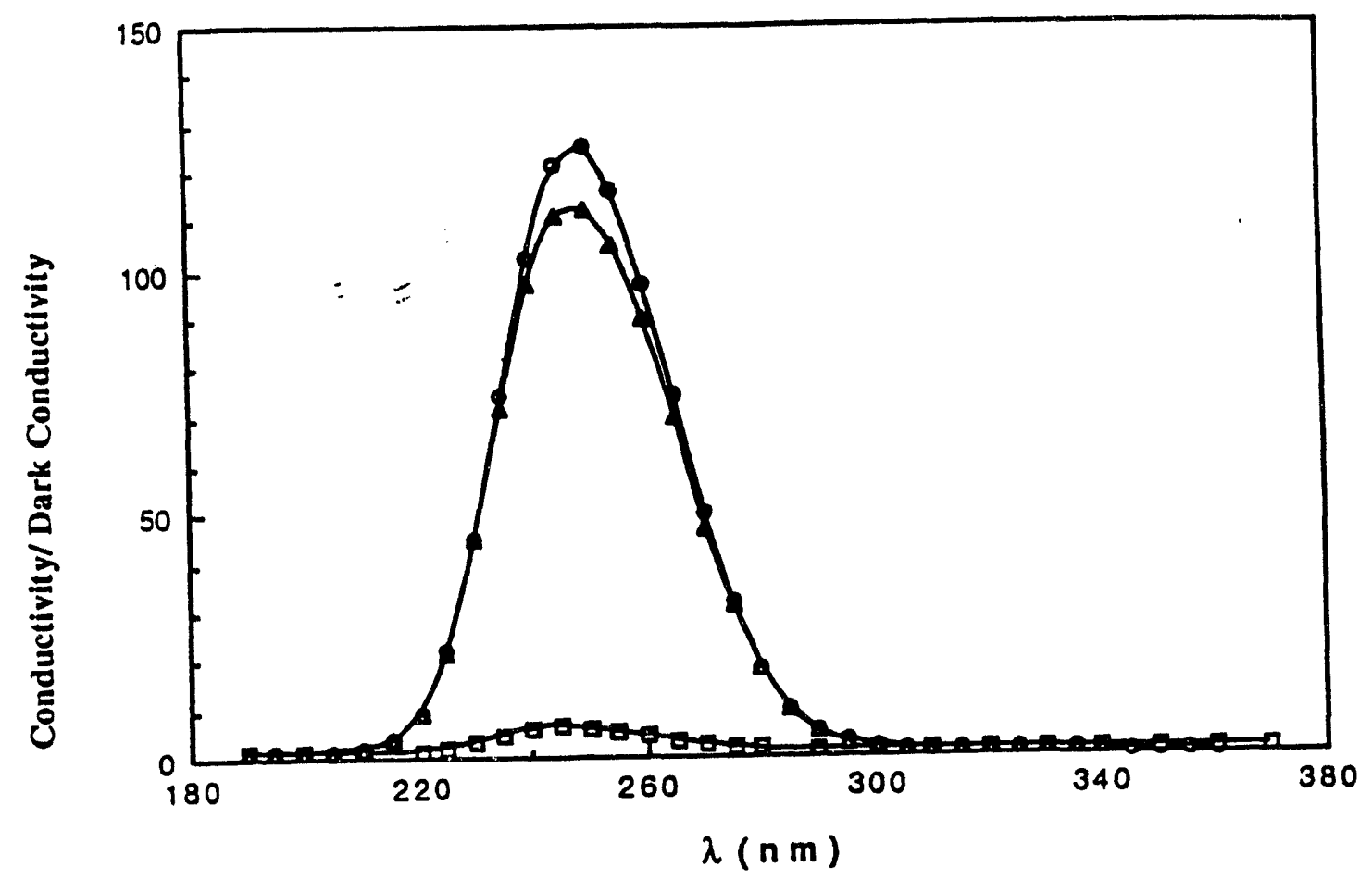

Circles: untreated

Triangles: acid treated for $8 \mathrm{hr}$ at $70 \mathrm{C}$

Squares: delignified for $1 / 2 \mathrm{hr}$ with acid chlorite

Figure 2. The wavelength dependence of photoconductivity of longitudinal sections of Douglas fir 30 micrometers in thickness.

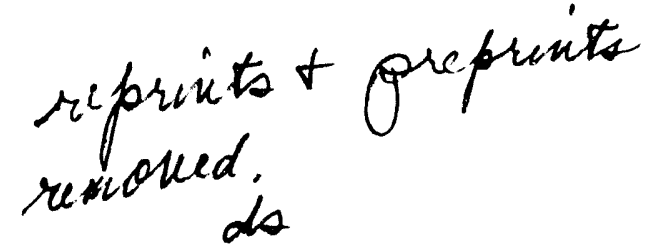




\section{0}

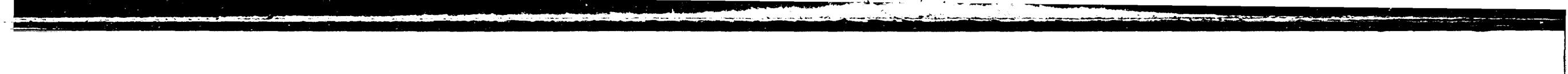

